\title{
Profesionales en red: acción colectiva, construcción y deconstrucción de ideas y prácticas. Entrevista a la Red de Psicólogxs Feministas - La
}

\section{Plata}

\author{
Clara Maidana \\ Universidad Nacional de La Plata, Argentina \\ clara.maidanagorlero@gmail.com \\ Gisela Manzoni \\ Universidad Nacional de La Plata, Argentina \\ giyitan@yahoo.com.ar \\ Paula Soza Rossi \\ Universidad Nacional de La Plata, Argentina \\ paulasoza11@yahoo.com.ar
}

En los últimos años, la masificación de los debates en torno a la violencia de género, la diversidad y el feminismo están haciendo eclosión e impactando en la formación y práctica de lxs distintos profesionales. Si bien no se consolidó aún una formación transversal e integral con perspectiva feminista en las universidades argentinas, se están fortaleciendo antiguos espacios y generando otros nuevos, como la Red de Psicólogxs Feministas, en los que lxs profesionales, lxs psicólogxs en particular, se especializan en abordajes que parten desde esta mirada.

La desnaturalización de la violencia de género y de la opresión cisheteropatriarcal, así como los debates públicos sobre la legalización del aborto, generan nuevas preguntas y reflexiones en las personas y promueven una historización de la propia vida que descubre experiencias invisibilizadas. En este sentido, espacios como los de la Red, habilitan la posibilidad de trabajar estas demandas y necesidades subjetivas y culturales.

La siguiente entrevista fue realizada por el equipo de la sección homónima de la revista Descentrada ${ }^{1}$ a dos profesionales de la Red, Lucia Pérez Muzzin y Lorena Schwab, en su consultorio ubicado en la ciudad de La Plata. La Red de Psicólogxs Feministas es una asociación de profesionales nacida en el año 2016. Desde sus inicios hasta la actualidad, creció exponencialmente, y hoy se ubica en distintos lugares del país: en la Ciudad y la Provincia de Buenos Aires (GBA, Tandil, Bahía Blanca), La Plata, la Costa Atlántica (Mar del Plata), Córdoba, Mendoza, San Luis, La Rioja, Ushuaia, Río Negro y Santa Fe. Conformada por, aproximadamente, 250 profesionales, la Asociación concibe de forma integral la salud mental, desde una mirada que incluye los aportes de los estudios de género, la teoría queer, los estudios trans y sobre masculinidades. En particular, se incorporan las reflexiones de los avances de los estudios de género a la psicología y el psicoanálisis. Esta amplitud contempla, a la hora de trabajar sobre los padecimientos psíquicos, la existencia de relaciones de poder asimétricas entre los géneros, las identidades y las sexualidades. De esta mirada integral se desprenden las tres funciones básicas de la Asociación: la atención, la formación y la difusión de campañas de sensibilización.

El diálogo con las dos profesionales busca presentar la forma y las particularidades de la Red, su historia y organización. A lo largo de la entrevista, ellas desarrollaron algunas de las aristas principales de la mirada psicoanalítica que prevalece en sus consultorios y la modalidad de articulación de las teorías psicoanalíticas con la praxis y la ética feminista. Además, comentaron cuál es el efecto que produce la evidencia de que el espacio y lxs profesionales son feministas, cómo esto habilita hablar de cuestiones que fueron, durante muchos 
años, silenciadas. Parte de la demanda que llega a la Red se relaciona con las huellas que marcan a lxs sujetxs en previas experiencias psicoanalíticas no feministas, muchas veces expulsivas. Las profesionales se refirieron, también, a los indicios del sistema cisheteropatriarcal en la configuración psíquica, en las inquietudes y en las vivencias de lxs sujetxs que asisten a terapia en el marco de la Red.

E - ¿Cuándo surge la Red y quiénes la integran?

PF - La red surge en mayo de 2016, en la Ciudad Autónoma de Buenos Aires (CABA). Cuando comenzó, se designaron cinco personas como coordinadoras que realizaron una convocatoria amplia para que se sumen profesionales. Yo me sumé a partir de esa convocatoria. La Plata fue uno de los primeros lugares, por fuera de $\mathrm{CABA}$, que se consolidó. $\mathrm{Al}$ inicio, fuimos cuatro o cinco, pero se incorporó gente rápidamente debido a la alta demanda de atención.

Actualmente, somos 32 profesionales en La Plata y, en toda la red, un total de 250 integrantxs. Con el tiempo, se fueron abriendo regionales en diferentes puntos del país: en la provincia de Buenos Aires, en Mar del Plata, Tandil, Bahía Blanca, Zona Norte, Conurbano, Rosario, Santa Fe, Córdoba, Mendoza, La Rioja, San Luis, Bariloche y Ushuaia. La sede más grande sigue siendo CABA, lógicamente.

E - ¿Cómo fue su acercamiento a la Red?

PF - Yo conocía a una de las coordinadoras, vi una publicación en la que invitaban a ser parte y me acerqué. Éramos pocas en ese momento. El primer encuentro fue una entrevista descontracturada. Hablamos sobre cómo funcionaba y cómo era el trabajo en la Red, y compartimos cuál era nuestra formación. Al principio, las derivaciones se realizaban desde CABA, el proceso estaba centralizado. Después, a medida que se fueron incorporando profesionales en La Plata, la dinámica fue cambiando.

E - ¿Cómo surge el nombre de la Red?

PF - Lo eligieron en Capital Federal, desde el grupo de coordinación inicial. Ellxs venían de otras experiencias, de trabajar en conjunto en otros espacios. Lxs profesionales, al encontrarse, develaron que en la práctica privada muchas personas compartían sus malas experiencias al asistir a espacios de atención expulsivos, donde se habían sentido discriminadxs o violentadxs de alguna forma. A partir de este encuentro, vieron la necesidad de trabajar desde la perspectiva feminista.

También, en la lectura que se realizaba de la coyuntura y de la masificación del feminismo, nació la necesidad por parte de lxs mismxs profesionales, de agruparse. En principio, surgió la idea de que la Red sea más amplia, no sólo psicólogxs, sino que sea de profesionales de atención de la salud en general. Sin embargo, en la práctica, resultó complejo pensar en llevar adelante esa interdisciplinariedad. Por este motivo, se definió que sea de psicología en particular.

PF - Yo me inserté a partir de la referencia de una compañera de mi espacio de militancia, como sucedió en muchos casos. Al incorporarme, tuve una especie de entrevista grupal, en Capital Federal, con las personas de la coordinación. Había mandado mi currículum previamente y, tiempo después, me llamaron.

Actualmente, es un poco más personalizado el ingreso. A medida que la Red crecía se fue modificando y puliendo el proceso de ingreso, en pos de construir un marco de seriedad profesional. Existe una persona que se encarga del área de recursos humanos y recibe currículums. Otra persona se encarga, acá, en La Plata, de hacer entrevistas, como en cualquier trabajo.

En general, las recomendaciones de ingresos las hacemos las mismas profesionales de la Red, porque hay gente que escribe como bolsa de trabajo. El objetivo es que no funcione únicamente como una posibilidad laboral, sino que haya profesionales que tengan formación y se especialicen en la perspectiva de trabajo acordada.

E - En torno al nombre de la red, con lenguaje inclusivo ¿ ¿tuvieron inconvenientes?

PF - Somos una Asociación Civil y tenemos personería jurídica hace un año. Fue toda una disputa legal que acepten la nominación, el hecho de que sea en lenguaje inclusivo, con $\mathrm{x}$.

$\mathrm{E}-¿$ Molestó solo la x? 
PF - No podemos afirmar cuál fue la interna. Quizás, también fueron otras cosas. Para el Colegio de Psicólogos es nuevo que haya una asociación, creo que debemos ser las primeras en organizarnos como red. Las normas de atención no están pensadas para esas identidades colectivas. Eso también nos trae otras obligaciones. Tenemos que ser cuidadosas porque representamos a una Asociación Civil.

E $-_{i}$ Existen criterios acordados para ser parte, además de tener voluntad de entrar, y estar buscando inserción laboral?

PF - Un acuerdo básico es que la persona tenga algún tipo de formación específica en cuestiones de género y feminismo, y experiencia clínica. Hay personas con trayectoria académica en el tema, que hicieron posgrados, especializaciones, y personas que hemos tenido un recorrido por fuera de la academia, en el plano del activismo y la militancia. La formación es diversa. Además, tenemos un protocolo donde están pautadas algunas cuestiones acerca del funcionamiento de la Red con las que tenemos que estar de acuerdo. Por ejemplo, tenemos criterios políticos comunes en torno a la posición respecto a la legislación del aborto, al respeto por las leyes de identidad de género y de violencia de género, y siempre, ante todo, creer en los testimonios de las personas.

E - ¿Cómo definirían a la Red de Psicólogxs Feministas?

PF -Es un espacio de encuentro, construcción, acompañamiento, en el cual podemos pensar y construir una articulación compleja: la de la psicología en términos amplios, con variables en su interior, el ejercicio profesional como psicólogas y la posición ideológica, política y ética feminista.

Asimismo, es un espacio de formación que se enriquece de los distintos recorridos que traemos, desde la militancia, desde la academia, desde distintas corrientes de la psicología y sus revisiones críticas. En La Plata, como la mayoría nos conocemos de otros espacios de sociabilidad, tenemos mucho más contacto y cercanía. Eso potencia el trabajo y fortalece la Red.

E - ¿Es un espacio de experiencia colectiva pero también de sostén?

PF - Si, es de sostén, ya que no nos dedicamos únicamente a la práctica clínica privada, que es un ejercicio que puede ser muy solitario. La red es un espacio de intercambios y de acompañamiento entre lxs profesionales.

E - ¿Qué identidades y franjas etarias hay en su composición?

PF - Por lo general, la franja etaria abarca personas que están iniciando hace pocos años su trayectoria profesional. En CABA, hay personas que ya tienen recorridos más largos. Por otro lado, quienes somos parte de la Red no solamente somos psicólogas que hacemos terapia en el consultorio, hay personas que son una referencia dentro de la red porque se dedican a dar capacitaciones y formación, hacia adentro y hacia afuera de la asociación.

En torno a las identidades de quiénes la integran, en La Plata somos mayoritariamente mujeres y lesbianas, hay un solo varón. En CABA y el resto de los lugares del país, hay más varones. En la Red en general, los varones son muy pocos. Esto, sin embargo, es una característica propia de la psicología (constitución de sus egresados) en la actualidad. En La Plata, es un tema sobre el que venimos pensando, ya que, hay personas que desean atenderse con profesional varón.

E - En La Plata, ¿son todxs egresadxs de la UNLP?

PF -No, actualmente tenemos tres compañeras egresadas de otras universidades.

E - Es decir que, ¿existen distintas vertientes respecto a la teoría del psicoanálisis hacia el interior?

PF - En La Plata, somos la mayoría psicoanalistas. Dentro del psicoanálisis, tenemos nuestras variaciones. Las corrientes a las que adscribimos lxs profesionales de la Red son diversas. Tenemos una sola compañera que hace Terapias Cognitivas Conductuales (TCC). En Capital, hay más profesionales que hacen cognitivas conductuales o Gestalt. ${ }^{2}$

E - ¿Tienen algún acuerdo sobre la práctica de la escucha?, ¿es un abordaje diferente al del psicoanálisis clásico? 
PF - Los acuerdos que tenemos son los que nos unen como feministas. Por ejemplo, en torno al respeto de las identidades autopercibidas, al acompañamiento en las situaciones de violencia. Asimismo, entendemos al patriarcado, al cisexismo y a la heterosexualidad obligatoria como variables que producen un padecimiento psíquico específico. La forma de abordaje de este padecimiento puede diferir de acuerdo a las corrientes en las que cada profesional se ubica dentro de la psicología.

PF - Acordamos realizar un abordaje que no sea revictimizante, sino respetuoso y libre de prejuicios. Como profesionales no nos dedicamos exclusivamente a situaciones de violencia de género, sino que nuestro trabajo implica una mirada de carácter integral.

E - ¿Cómo funciona la organización de la Red?

PF - Fue cambiando a medida que la Red crecía. Al comienzo, se realizaban reuniones bimestrales en Capital Federal hasta que, en La Plata, comenzamos a consolidarnos como grupo. Entonces, nos pareció más productivo para nosotras encontrarnos acá, ya que teníamos problemáticas particulares. Esto permitió empezar a construir una identidad local propia, para atender cuestiones ajenas a la Capital Federal. Existen "delegadas" que funcionan como nexo con CABA, van a las reuniones y realizan un intercambio de propuestas. También se designaron a otras cinco personas que coordinan distintas áreas, ya que hay diversas tareas en torno a la personería jurídica y la contaduría, entre otras. Además, estamos implementando una modalidad de supervisiones horizontales.

PF - A nivel local, construimos una estructura replicando la general del área de coordinación de CABA, en función de nuestro crecimiento y atendiendo a las necesidades que surgen. Por otro lado, hay dos personas que se encargan de hacer las entrevistas iniciales. Lxs usuarixs suelen contactarse con la Red por Facebook o por mail, en ese caso lxs atiende una persona de La Plata que realiza la entrevista inicial para relevar la demanda y hacer una derivación adecuada y responsable, conociendo las posibilidades de cada profesional, su disponibilidad para brindar atención, teniendo en cuenta particularidades del usuarix, como la ubicación residencial o, por ejemplo, si su consulta refiere a un caso que está judicializado.

E - ¿Qué sería un caso judicializado?

PF - Puede ser un caso de abuso o de violencia, que está en manos de la justicia. Dependiendo de la particularidad, algunas consultas decidimos derivarlas a una institución estatal o a cooperativas. Trabajamos en red con otrxs profesionales que habitan distintas instituciones, en pos de ejercer una derivación responsable, ya que hay demandas que no podemos tomar.

E-Mencionaron la existencia de otros espacios de la Red en distintos puntos del país. ¿ Tienen interacción con ellos o sólo con Capital Federal?

PF - Hace poco tiempo, una compañera de Bariloche armó un área que se llamó "de articulación provincial", con el objetivo de generar intercambios que no estén mediados por CABA. Es algo reciente. Nosotras usamos una plataforma que se llama "Slak" y a través de ahí se tratan de generar encuentros.

E - ¿Con qué otras instituciones o espacios se vincula la Red?

PF - Hemos trabajado con organizaciones de abogadxs (como La Ciega), con sindicatos ligados a la salud, instituciones educativas, centros vecinales, cárceles, ONG como "Creando Lazos", para pacientes oncológicos.

E - Además de su trabajo de psicólogas, ¿trabajan de otras cosas?, ¿hay alguien que se dedique exclusivamente a la Red?

PF - Es una elección personal, hay quienes trabajan exclusivamente y quienes tienen otros empleos. En torno a la retribución, es importante no perder de vista que este es nuestro trabajo. Por un lado, tener presente que no somos el Estado ni tenemos sus recursos y, por el otro, trabajamos desde un posicionamiento alejado del asistencialismo o paternalismo. Por eso, es importante encontrar modos de retribución. Si no es monetaria, es con pacientes que sean respetuosos del espacio, que trabajen por sí mismxs: ese es un intercambio que consideramos válido, aunque no es posible tener diez pacientes así porque no comés (risas).

E $-¿$ Consideran a la Red como un espacio de militancia? 
PF - Personalmente, me costó mucho porque tenía otro espacio de militancia, muy distinto. Fue difícil, particularmente, pensar en esta intersección entre trabajo y militancia. Apostamos a la psicología feminista, algo que hay que construir, que lleva tiempo por fuera de los tiempos del consultorio, e implica poner el cuerpo, en otras cosas. Por este motivo, ahora considero que sí, es un espacio de "militancia", aunque lo pondría entrecomillado todavía.

PF - Yo creo que es algo muy incipiente. La modalidad de reuniones mensuales que implementamos recientemente le otorga un carácter más orgánico y da ese tono de militancia. Coincido en que es algo que está aún en construcción. Lo podemos ir notando cuando nos organizamos para participar en otro tipo de actividades, por ejemplo, una movilización, un taller (ahora recibimos un pedido de capacitación de la Unidad Penitenciaria Número 9, realizamos articulaciones con distintos sindicatos). Algunas son remuneradas, otras no. Cobramos cuando se trata de empresas privadas o a instituciones que tienen partidas presupuestarias destinadas a estos fines. Nos han llegado pedidos de capacitación para empresas, de una ONG, o de algunos grandes sindicatos que tienen capital destinado a eso, y en ese caso las personas que integran la Red cobran por ese trabajo.

E - ¿Qué implica hacer una psicología feminista y ser una psicóloga feminista?

PF - Hacer psicología feminista implica tener en cuenta las desigualdades de género. Vivimos en una sociedad heteronormada, que basa su sistema social, político, económico y cultural en la heterosexualidad como la única forma válida de relacionarse sexo-afectivamente y de construir relaciones de parentesco; en una sociedad en la cual las instituciones públicas y privadas encarnan el sistema patriarcal. Este anclaje influye para la escucha, para ver, a su vez, entender cómo esto influye en la conformación de la subjetividad. Con respecto a las distintas corrientes de la psicología, acordamos distanciarnos del psicoanálisis ortodoxo, porque no podríamos tener esa escucha (adecuada), ya que entendemos que puede ser patologizante de las identidades disidentes.

PF - Incluso, en relación a la violencia, es particularmente victimizante, generando una escucha culpabilizante. Para hacer una psicología feminista, tenemos que tener en cuenta lo que nos pasa en la vida cotidiana, poner en tensión algunas teorías con las que nos formamos como personas y como profesionales. Para eso, el trabajo en red es fundamental. Si no fuese por la militancia u otros caminos que hemos elegido recorrer, fuera de la facultad, nuestra formación disciplinar no hubiera sido puesta en cuestión.

Implica tener en cuenta estas variables que producen un padecimiento psíquico específico, que están instaladas como mandatos, como destinos, y producen cierta rigidización en las personas y en las subjetividades. Además, producen sufrimiento. Lo que hacemos es trabajar en pos de abrir, de romper, de producir más libertad, más movimiento, de ayudar en ese tránsito a una vida más libre, placentera y saludable, en términos integrales.

PF - Ser una psicóloga feminista es hermoso, implica ponernos en tensión a nosotras mismas y a nuestra formación profesional. Tensionar esos enunciados que traemos me produce mucho placer en el consultorio.

E - ¿Cómo fue el proceso de revisión de su formación disciplinar?

PF -Es constante y complejo, ya que hay toda una epistemología que revisar, cuestiones teóricas y conceptuales. También, la modalidad de trabajo en la clínica. Tenemos una formación sumamente amplia y compleja. Sin embargo, poder realizar estos cruces, evitando que sean sólo abrochamientos, no es sencillo.

PF - No buscamos que sea, solamente, desechar algunos conceptos aprehendidos, sino repensar todo el tiempo esos enunciados teóricos y encontrarles nuevas posibilidades.

E - ¿Han podido armar una genealogía diferente a la formación clásica? ¿Tienen referentes?

PF - La autora que más ha pensado conceptos clásicos del psicoanálisis, en esta línea, que los ha trabajado y reformulado, es Silvia Bleichmar. ${ }^{3}$

PF - También lo hizo Ana María Fernández, Eva Giberti.

PF - Silvia era socióloga y propone reflexiones y cuestionamientos de una manera más integral. No solamente en cuestiones de género, ya que murió en 2007 y muchas cosas no estaban instaladas. Sin embargo, 
llevó a cabo cruces teóricos pensando en el impacto de lo social en la subjetividad y en la construcción de sujeto. Algunas de esas reflexiones pueden abordarse desde una lectura feminista, y reflexionar sobre el modo habitual de pensar la identidad sexual. Argentina tiene una tradición de psicoanálisis más amplia que otros países, también tiene grandes producciones de reflexión intelectual, fue pionera en este sentido. Ana María Fernández es una de las más reconocidas.

$\mathrm{E}-¿$ Cómo denominan a quienes les consultan?

PF -A mí, particularmente, "paciente" no me gusta, pero estoy acostumbrada y a veces lo uso, pero la palabra refiere a alguien que espera, que está en otro nivel, es propia del modelo médico hegemónico. "Consultante" podría ser.

PF -Usuario, usuaria, es una palabra más horizontal. La denominación es una de las huellas de la formación recibida, lastres que son difíciles de transformar.

$\mathrm{E}-¿$ Por qué creen que lxs usuarixs eligen profesionales feministas?

PF - Hay muchas personas que vienen de experiencias de atención previas, en las que fueron maltratadas, no fueron escuchadas, se sintieron juzgadas y, por lo tanto, fueron tratadas con prácticas expulsivas y poco contenedoras. Otras, no vienen de esos espacios, sino por la referencia a la perspectiva de trabajo de la Red, que les da ciertas garantías de seguridad y comodidad al llegar al espacio, habilitando la posibilidad de hablar más libremente que con profesionales con los que no tienen esa referencia previa.

PF -El feminismo, de un tiempo a esta parte, ha tenido mucho espacio en los medios de comunicación, esto hizo que más personas quieran acercarse, no necesariamente a una psicología feminista, pero sí a espacios de este tipo.

E - ¿Cómo es la recepción de la Red por parte de lxs demás profesionales?

PF - He escuchado críticas hacia nosotras, de otras profesionales que piensan que la red es una cuestión más ideológica, y que debería estar "más limpia” la atención y la escucha.

E - Un reclamo de objetividad...

PF -Sí.

PF - Nuestra formación tiene un sesgo lacaniano. Hay ciertas cuestiones que tienen que ver con la denominada "abstinencia" del analista, que implica un posicionamiento pasivo en la escucha, cierta opacidad. Es decir, que no tiene que haber nada de quién es el analista dentro del consultorio, porque eso podría llegar a interferir en lo que el usuarix dice dentro del espacio. Nosotras no coincidimos con eso. Primero, porque consideramos que eso también es un posicionamiento político y ético. Además, hemos comprobado en nuestro ejercicio profesional que, al decir abiertamente que somos feministas, al contrario de obturar, habilita y, muchas veces, facilita el trabajo.

PF - Una cosa que me parece importante aclarar es que tener presente cuestiones políticas y de lectura de lo social no implica perder de vista la singularidad al interior del consultorio. Eso hace que nuestro trabajo sea muy artesanal. No se trata, sin embargo, de que hagamos una "bajada" de todo esto que venimos diciendo en todxs los pacientes por igual, sería violento. Por eso esta articulación es un desafío complejo.

E - ¿Cuáles son las vulnerabilidades que produce el patriarcado que más se repiten a nivel intrapsíquico?

PF - Es difícil. Muchas veces aparecen cuestiones referidas a la sexualidad heteronormada, aunque no sé si puedo pensarlo como una vulnerabilidad. Pero, de alguna manera, es una restricción que te impone el sistema sobre tu propia vida. Aparece muchísimo en el consultorio la pregunta por cómo flexibilizar cuestiones referidas a la sexualidad, a los modos de vincularse (relaciones abiertas, poliamorosas, que se construyen por fuera de la monogamia y los mandatos del amor romántico, basadas en la creatividad a la hora de establecer acuerdos amorosos y con nuevos problemas al respecto). Sí aparecen, más claramente, cuestiones de violencia, de abuso infantil, experiencias que son resignificadas. Al trabajar con personas adultas, es posible identificar, en el proceso de historización, situaciones en su trayecto de vida que hayan sido abusivas o violentas.

PF - Cuando surgió la denuncia pública del Colectivo de Actrices Argentinas, puntualmente de Thelma Fardin, aparecieron un montón de consultas de pacientes que empezaban a identificar y reconocer este tipo 
de situaciones. Como consecuencia, hoy es más habitual que las experiencias de abuso se puedan hablar y compartir, y además se puedan denominar de esta forma, como abuso, no como antes que se naturalizaban. La cuestión del lugar de la mujer en general, no sé si enmarcarlo en "vulneraciones", pero sí es un tema que estaba naturalizado y se empieza a cuestionar.

$\mathrm{E}-\dot{ } \mathrm{A}$ Atienden a personas que han ejercido violencia?

PF - Algunas profesionales de la Red sí, otras no. En la Plata, hay compañeras que no atendemos, pero otras pueden trabajar con esas situaciones y decidieron que sí.

PF - A raíz de la explosión de los escraches, surgieron más consultas de varones, pensando en cuestionarse privilegios o revisar algunas actitudes. Ahí fue cuando revelamos quiénes querían trabajar con estas situaciones, para tenerlo claro en las derivaciones. Aunque no es tan habitual recibir consultas de varones que se relacionen en términos violentos o que hayan ejercido violencia, porque implica un cuestionamiento profundo de sí mismos y esto no es frecuente que suceda, sino el mundo sería otro.

PF - Si alguien va a consultar porque quiere hacer terapia por eso, ya hay varias que dijimos que no. Pero no todos dicen por qué consultan. Por otro lado, estas cuestiones pueden surgir después, en el análisis.

PF - Yo atiendo a un solo varón heterosexual. Se fue dando así, pero también hay cuestiones que me cuesta trabajar con el "ser varón".

PF - Yo trabajo con dos varones, uno de la Red y otro no.

$\mathrm{E}-¿$ Qué pasa cuando el ejercicio de la violencia aparece en otro tipo de identidad?

PF - La violencia en parejas homosexuales o lésbicas es un tema invisibilizado. Tenemos una compañera, Belén, de Capital, que se especializa en violencia en vínculos lésbicos. Personalmente, en el consultorio no han surgido situaciones de gravedad. Aparecen modos heteronormados de relacionarse que se replican en cualquier tipo de vínculo, violencias más sutiles como celos, control, desigualdades en lo que cada parte de la pareja puede hacer. En las configuraciones de parejas abiertas aparece mucho eso.

PF - Tuve un caso en el que una paciente se acercó porque su ex psicóloga le dijo que no existían las relaciones abiertas, o al menos esa fue la interpretación de la paciente. Pero en esto no hay una definición clara de lo que es "pareja abierta", por eso hay personas que buscan una definición. Está bueno tener presente que es un camino, saber que no hay una sola definición al respecto, sino que se da desde la singularidad de ese vínculo.

E - ¿Cómo aparece la problemática del aborto en usuarixs, tanto en relación a lo personal como en torno al debate social?

PF - Me han tocado muchas situaciones de pacientes que se han hecho abortos hace diez o quince años y que lo pueden decir recién ahora, en el consultorio, y que lo saben sólo otras dos personas más de su entorno, por ejemplo. Tengo el caso de una paciente que no se anima a decirle a su ginecóloga que se hizo un aborto. Otros llegan y dicen: "Hice terapia muchos años y nunca lo dije, pero ahora te digo que me hice un aborto hace 10 años".

PF — La referencia previa de ser feminista habilita eso.

PF - Pero, en general, no hay ningún sufrimiento detrás de eso, nunca más vuelven a mencionarlo. Parece que es algo que ha causado una situación traumática pero después no vuelve a aparecer. Lo dicen por si durante la terapia nosotras lo relacionamos con algo que las pacientes nos puedan llegar a contar.

PF - Aparece de formas diversas. Hay casos de personas que atravesaron un aborto solas, y fue doloroso y, de alguna forma, traumático. Otras personas lo cuentan como algo que sucedió y no hay ningún padecimiento puntual. También, aparece el tema cuándo están acompañando a una amiga, que quizás no va a terapia. El debate político también surge, por ejemplo, con pacientes que comentan: "Fui con mi mamá, y ella está en contra". Es decir, como debate en los enfrentamientos en la familia, amigos, lugares de trabajo. Es un indicador.

PF - Pero en relación a las personas que consultan, hay situaciones muy diversas, no es que sea traumático en sí, sino que lo traumático es enfrentarlo solas, o la clandestinidad que implica estar detrás de una mentira. No me ha pasado que alguien venga a terapia por esto puntualmente. 
E $-¿$ Han recibido personas que lleguen al consultorio por conflictos en torno a su identidad sexual?

PF - Si (todas).

PF - También, suelen llegar situaciones de violencia de género como motivo de consulta.

E - ¿Qué porcentaje de lxs usuarixs provienen de sectores sociales más vulnerables?

PF - Muy pocos.

PF - Que consulten a la red directamente pocos, sí tal vez de derivaciones que hacemos de personas del bachillerato de educación popular para jóvenes y adultos, se piensa si pueden pagar o no, o pensamos alguna alternativa para derivar en algún lugar público.

PF - Aparecen más demandas de intervención con modalidades de capacitaciones, talleres, y acompañamiento.

E - ¿Cómo aparece el vínculo entre la militancia y la agenda feminista con lxs consultantes?

PF - Impacta de formas diversas. A veces con preguntas como "fui a una marcha, fui a una charla, y me hizo pensar en algo de la propia historia”. Atraviesa también a partir de los vínculos que les consultantes tienen con sus amistades, con los lugares por los que transitan, con sus familias.

PF -Especialmente en las personas más jóvenes. En principio, cuando se formó la Red, llegaban muchas consultas de personas que militaban en alguna organización. Ahora no es tan frecuente, las consultas son mucho más diversas.

Durante el diálogo, las entrevistadas destacaron la experiencia particular de ejercer su profesión de psicólogas en la Red y fundamentalmente, la importancia medular de la reflexión continua, al momento de contemplar y reconocer los efectos de la cultura patriarcal sobre las subjetividades y el consecuente sufrimiento psíquico en las personas.

Cuando desde el perfil editorial, al inicio de la entrevista, hablamos del crecimiento y la fuerza del movimiento feminista, nos referimos también a este tipo de espacios alternativos como lo es la Red de Psicólogxs Feministas.

Esta Red es fruto de las necesidades de nuestro tiempo, del compromiso y la responsabilidad de "estar a la altura de las circunstancias" y de responder a las demandas de equidad. Es decir, para las entrevistadas, es fundamental acompañar a lxs usuarixs para revisar las construcciones identitarias, los mandatos sociales de género y su influencia en sus decisiones vitales; como también para acompañarse entre sí, a la hora de poder aunarse, reflexionar, especializarse, compartir y construir una perspectiva integral y feminista de la Salud Mental. Sin duda, apuestan a una modalidad de trabajo y atención adecuada y pertinente a las demandas de lxs usuarixs donde desde el respeto a la diversidad humana, intentan desarmar constructos teóricos anquilosados, prácticas profesionales naturalizadas que no sólo reproducen discriminaciones y desigualdades, sino que además revictimizan sin reconocer y atender adecuadamente un sufrimiento psíquico, que culpabiliza a lxs sujetxs y no conmueve las estructuras sociales de las desigualdades de género.

Para contactarse con la Red de Psicologxs Feministas www.redpsicologxsfeministas.com o redprofesionalesfeministas@gmail.com

\section{Notas}

1 Agradecemos la colaboración y las sugerencias realizadas al guion de la entrevista por parte de lxs psicólogxs Adriana Beatriz Rodríguez Durán, Ariel Martínez, integrantes del CINIG - FaHCE-UNLP, y Carolina Carrizo de la cooperativa de salud mental TRAMA. Asimismo, agradecemos la colaboración en la escritura de las notas ampliatorias a Adriana Beatriz Rodríguez Durán y Ariel Martínez.

2 Psicoanálisis: Existe consenso en delimitar el surgimiento del psicoanálisis en los inicios del siglo XX cuando Sigmund Freud publica La interpretación de los sueños. Allí comienzan a cobrar forma algunos de los principales aportes conceptuales, tales como la idea de una instancia psíquica Inconsciente que, como un genuino sistema, funciona con 
una legalidad propia y produce efectos susceptibles de ser interpretados. Este corpus teórico naciente, muy disruptivo con la tradición médico-psiquiátrica imperante en el momento, fue instalándose progresivamente en la intelectualidad europea. Un rasgo peculiar a destacar es la inmediata institucionalización del psicoanálisis, lo cual agregó al prolífico despliegue de ideas un potente atravesamiento político-institucional que estableció claramente qué ideas y qué analistas pertenecía de pleno derecho a las filas del psicoanálisis. La ortodoxia psicoanalítica se configura, de este modo, en un conjunto de elementos conceptuales altamente salvaguardadas por un conjunto de instituciones que se arrogan la certeza de dictaminar cuál es el psicoanálisis que sigue, legítimamente, el dictum de su padre fundador. Actualmente, marcos epistemológicos y políticos emergentes desde el pensamiento feminista y disidente nos permite observar el carácter universal y a-histórico que envuelve aquellos aportes. Tal es así, que existen fuertes críticas a los sesgos falocéntricos, sexistas y misóginos que la teoría conserva, aún en conceptos fundamentales, como el Complejo de Edipo. Cabe destacar que, a pesar de las críticas, numerosas intelectuales feministas rescatan la potencia de un conjunto de herramientas conceptuales propias del psicoanálisis. Desde allí han optado por asumir el desafío de delinear un psicoanálisis sumamente heterodoxo que entiende cómo el Patriarcado y la Heterosexualidad obligatoria configuran órdenes políticos no ajenos a la constitución subjetiva. Por ende, enfrenta el desafío de pensar un psicoanálisis en contexto que aborda el padecimiento subjetivo producto de diferentes ejes de poder socio-históricamente delineados.

Tradicionalmente suele ordenarse el psicoanálisis posfreudiano en dos grandes vertientes, el psicoanálisis lacaniano, por un lado, y el psicoanálisis de las relaciones objetales por otro. El pensamiento lacaniano ha sido fuertemente criticado, aunque también sumamente rescatado, por pensadoras feministas europeas que organizan sus ideas en torno a la categoría de Diferencia Sexual. El Psicoanálisis de las relaciones objetales ha sido una potente plataforma conceptual que ha permitido al feminismo norteamericano articular psicoanálisis con la categoría de Género. Aun así, estas líneas psicoanalíticas son el producto de un criterio que simplifica la cuestión. Existen multiplicidad de derivas, incluso dentro mismo de las vertientes destacadas. Como fuere, interesa destacar que el reconocimiento de cuáles de ellas pertenecen al verdadero psicoanálisis responde a la cristalización de poder que se libra dentro de las instituciones psicoanalíticas que concentran la legitimidad.

Terapia Gestáltica: Se fundamenta en la psicología de la Gestalt, el existencialismo y la fenomenología. El psiquiatra psicoanalista Friedrich Perls y la psicóloga Laura Ponser fueron lxs iniciadorxs de la psicoterapia gestáltica $(1942,1966)$. Aunque se reconoce al sociólogo Paul Goodman y al psicólogo Ralph Hefferline (1951) como los que establecieron sus bases fundamentales. Es una terapia holística, que considera a la persona como una totalidad, abordando todas las áreas (cognitiva, emocional, corporal, relacional, espiritual). Se centra en el "aquí y ahora”. Su método principal es potenciar el "darse cuenta", acrecentando la conciencia de lo que se percibe, siente y actúa. La terapia gestáltica intenta proporcionar herramientas para ampliar la toma de conciencia, con el fin de clarificar problemas o confusiones, ayudando a descubrir respuestas, que permitan restablecer el equilibrio homeostático. El/la terapeuta facilita el proceso realizando observaciones fenomenológicas y proponiendo técnicas para incrementar el darse cuenta de lxs usuarixs.

Terapias Cognitivas-Conductuales (TCC): modelo de psicoterapia breve, centrada en el presente. Los fundamentos teóricos son el conductismo y la psicología cognitiva, y los filosóficos provienen de la filosofía de los estoicos, quienes confiaban en la razón como medio para dominar las emociones e instaban a no dejarse perturbar por los hechos, sino someterlos a juicio. Un principio básico de las TCC es que los determinantes de nuestra conducta no son las circunstancias en sí mismas, sino el significado que le atribuimos a estas. El tratamiento se caracteriza por ser directivo, estructurado y en un tiempo limitado. Emplea tanto técnicas conductuales como cognitivas con el objetivo de identificar pensamientos, creencias y conductas problemáticas, para realizar una reestructuración de creencias que permita a la persona percibir de manera más realista las situaciones y modificar sus esquemas desadaptativos productores de síntomas. Representantes principales: el psicólogo clínico Albert Ellis (1962) el psiquiatra Aaron Beck (1967).

3 Silvia Bleichmar (1944-2007) Doctora en Psicoanálisis por la Universidad de París VII (tesis dirigida por Jean Laplanche) Psicóloga y Socióloga por la Universidad de Buenos Aires. Docente en las universidades nacionales (de Buenos Aires, La Plata y Córdoba) e internacionales de México, Francia, Brasil y España. Sus obras más conocidas son: En los orígenes del sujeto psíquico (1986), La Fundación de lo Inconsciente (1993), Clínica psicoanalítica y neogénesis(1999),Dolor pais(2002), La subjetividad en riesgo(2005), Paradojas de la sexualidad masculina (2006), No me bubiera gustado morir en los '9 (2006). Fue galardonada con el Premio Konex de platino (2006) y fue distinguida como Ciudadana Ilustre de la Ciudad de Buenos Aires (2007).

Ana María Fernández. Doctora en Psicología (UBA). Psicoanalista (UNLP) Analista Institucional. Profesora Consulta e Investigadora de la UBA. Titular de Teoría y Técnicas de Grupos e Introducción a los Estudios de Género en la Facultad de Psicología, UBA. Desde 2003 es Profesora Plenaria. Dirige investigaciones en la UBACyT desde 1986. Dicta posgrados en diversas universidades nacionales, tales como el Programa de Actualización en el Campo de problemas de la subjetividad (UBA). Invitada en universidades extranjeras: EEUU, Francia, España, Italia, México, Colombia. Autora de numerosos libros, entre ellos: El campo grupal (1986), La mujer de la ilusión (1993), Instituciones Estalladas (1999), Politica y Subjetividad (2006), Las lógicas colectivas (2007), Las lógicas sexuales: amor, política y violencias (2009), La diferencia desquiciada (en coautoría con William Siqueira Peres) (2013), Jóvenes de vidas grises (2013). Premio Naciones 
Unidas/Mujer (2000) y Premio Konex de platino (2016). Fue condecorada con el título de Graduada Ilustre de UNLP, entre otras distinciones.

Eva Giberti. Dra. honoris causa en Psicología por la Universidad Nacional de Rosario, (2006) ypor la Universidad Autónoma Nacional de Entre Ríos, (2010). Lic. en Psicología y Asistente social (UBA). Fundadora de la primera Escuela para Padres de Argentina en el año 1957. Asesora del Registro Único de Aspirantes a Guarda con fines adoptivos de la CABA. Desde 2006 coordina el Programa Víctimas contra las Violencias (Ministerio de Justicia y Derechos Humanos de Nación). Ex Docente de la Universidad de Buenos Aires (UBA) en la Especialización de Posgrado en Violencia Familiar y en la Especialización en Psicología Forense en la Universidad de Ciencias Sociales y Empresariales (UCES). Entre sus libros, destacamos: La familia a pesar de todo (2005), Adopción Siglo XXI (2010) Incesto paterno filial (2014), Abuso Sexual contra niños y niñas (2015), Mujeres y violencias (2017). Recibió el Premio Konex de Platino, año 2016. Ciudadana ilustre de la Ciudad Autónoma de Buenos Aires, desde el año 2003. 